\title{
When should care be withdrawn?
}

\author{
Saad Farooqi MD, Michael Nugent MA, Gilbert Berdine MD
}

$C_{A S E}$

Hospital Day 1: A 55-year-old man with chronic back pain was brought to the hospital by EMS after a cardiac arrest. The patient's brother stated that the patient had been drinking excessive amounts of alcohol and ingesting large quantities of oxycodone for the past 2-3 months. He was brought in to the Emergency Department approximately 20 minutes of ACLS in the field and started on a cooling protocol. The patient had continuous blinking and vertical eye movements, and an EEG showed status epilepticus. The patient was started on a propofol infusion, and the neurology service was consulted. Continuous EEG monitoring showed epileptiform activity; a MRI of the brain was consistent with anoxic brain injury. His neurological examination demonstrated intact brain stem reflexes. The family inquired about the patient's prognosis and were told that meaningful recovery was unlikely but that his prognosis could not be determined until his seizures were controlled and the sedatives were discontinued. The family wanted to withdraw care, but after the neurology consultant reiterated to them that we did not have sufficient data for a prognosis they agreed to observe the patient for 24 hours.

Hospital Day 2: The next day we were informed that the patient's wife -- with whom we had spoken only over the phone -- wished to stop all medications and remove him from life support because the patient's wishes were to not be kept on life support. After several discussions we followed her wishes and discontinued all medications except those necessary for comfort. The patient was extubated using a termination of care protocol. Following extubation, sponta-

Corresponding author: Gilbert Berdine, MD Contact Information: gilbert.berdine@ttuhsc.edu DOI: 10.12746/swrccc2013.0205.054 neous respiration and vital signs were adequate without further life support.

Hospital DaY 3: The following morning the patient showed significant neurological improvement; he was opening his eyes, recognizing family members, following commands, moving all extremities, and even vocalizing with a few words. Due to the change in clinical status the family decided to increase the level of support to maintain hydration and electrolytes and consider nutrition. Later that day his mental status again deteriorated, continuous EEG showed seizurelike activity, and anti-epileptic medications were restarted. He then developed respiratory distress with significant upper airway secretions, $\mathrm{O}_{2}$ saturations in the $70 \mathrm{~s}$, and respiratory rates in $50 \mathrm{~s}$. The DNR/DNI status was resumed per family's wishes. His level of care was decreased to comfort measures only so that his respiratory distress and air hunger could be adequately treated.

Hospital Day 4: The next morning he once again improved. He was more alert and making some effort to respond to commands. Once again his clinical status was reviewed with the family, and we increased some aspects of care, including routine labs and intravenous fluids with dextrose and multivitamins. That afternoon he became very agitated and received multiple doses of lorazepam, furosemide, and fentanyl which had little effect.

Hospital Day 5: The following day he was in severe distress and was not responding to our commands. We started him on a fentanyl infusion and consulted the palliative care service to provide symptomatic care and transitional planning. This service agreed with fentanyl infusion and added a lorazepam infusion. The patient was subsequently transferred out of the MICU to an inpatient hospice facility per the family's wishes. 


\section{Discussion}

Some health care workers involved in this case thought that the family wanted to withdraw care too soon and that the provision of more consistent supportive care and treatment of neurological and respiratory problems might have resulted in a better outcome. This case presented a difficult situation to the family and healthcare workers and can be analyzed from legal, ethical, religious, and philosophical perspectives.

\section{ETHICAL VIEWPOINT}

The conflict (or potential conflict) in this case is that there exists a treatment that MIGHT improve outcome. The physicians believe the therapy should be tried, but the family believes that their loved one would opt for withdrawal of further resuscitation. How should this conflict be resolved?

Consider the hypothetical situation in which we have a magic wand that grants 15 minutes of lucid conversation with the patient. During those 15 minutes, the health care team could present its case for treatment, but everyone would agree that the patient's decision on the matter would be final and would be honored by the health care team. The ethical approach to this problem becomes a determination of what the patient would decide, given the circumstances, rather than a determination of what would be 'best' for the patient. We must understand that 'best' is subjective rather than objective and the health care team must not project its own biases about the decision onto the patient or the family.

The simplest resolution to the problem occurs when someone has been granted medical power of attorney. The patient, in that situation, has decided to trust the judgment of the person to whom the power was granted in the event the patient cannot make decisions. All the health care team can do in this situation is make a pitch in favor of treatment, but the team would ethically be obligated to respect the decision of the power of attorney as final - just as it would if the decision were made by the patient.
The process becomes murkier when nobody has medical power of attorney. The family members may not be united in their opinion. The family may disagree with medical advice. Conflicts of interest may exist. All of these possibilities may complicate the decision making process and lead to uncertainty over who is in charge.

The ethical approach, as was the case above, is to determine what the patient would decide rather than what is 'best' for the patient. The usual and customary practice is that family is in a better position to determine what the patient would decide than a health care worker. If a family member emerges as the 'voice' of the family, that 'voice' should be honored by the health care team unless extraordinary circumstances exist.

A worse situation happens when multiple factions exist within the family. For example, one faction may agree with trying anticonvulsants, while another faction may insist on withdrawal of care. In this case, it is impossible to please everyone. The ethical approach would be to make a best effort of persuasion in favor of treatment, but to instruct the family members to discuss the matter and make a final decision among them. If the family is unable to unite on a single decision, then a court will have to appoint a medical guardian to resolve the conflict. In this case, however, the health care team is not the cause for the conflict and will not be blamed by the family.

The worst situation occurs when conflict of interest exists. A family member may stand to inherit a lot of property upon the death of the patient. Family members may regard expensive health care measures as needless dissipation of the estate. If the health care team believes the family is acting out of self-interest rather than in a good faith determination of what the patient would decide, the ethical decision would be to ask a court to appoint a medical guardian.

\section{LEGAL VIEWPOINT}

The above ethical analysis is based on patient 
autonomy. The Law, in our society, may or may not agree with the above analysis. Texas courts would usually apply the above ethical analysis to resolving disputes like this one. The Court might appoint a guardian and bind everyone involved to the decisions of the guardian. Less likely, the Court would act as the guardian and render judgment directly.

Other courts might not apply the above analysis to the decision. The California Supreme Court applied the principle of parens patriae, which is Latin meaning parent of the nation, when the Court decided the landmark case of Wendland v. Wendland. The Court required the guardian or conservator to act in the best interests of the patient rather than according to the expressed wishes of the patient. The doctrine of parens patriae dates back to sixteenth century decisions by the King's Bench court in cases involving non compos mentis adults (adults not of sound mind). This doctrine is generally applied in all U.S. jurisdictions when the patient is a child or chronically disabled such that the patient could never have expressed an adult statement of his or her wishes. Other courts have not followed the precedent of Wendland v. Wendland and continue to apply the above ethical reasoning for adults who have expressed competent wishes.

So, in the event in which a united family disagrees with medical advice, the health care team can request a court to appoint a medical guardian. This step should not be taken lightly. In the case under consideration, further treatment of the seizures had an uncertain outcome, and the most likely outcome was a poor one regardless of treatment. Taking legal steps to force an uncertain therapy with long odds for success is likely to lead to lasting bitterness between the family and the health care team. Furthermore, it is doubtful (absent extraordinary conflict of interest) that a Texas court would overrule the family on this matter. It is likely that legal steps would be viewed by family members as an unnecessary delay in the final withdrawal of care that only prolonged suffering by their loved one. While the health care team might prevail in a legal dispute, the bitterness generated by the legal conflict would complicate any further care.

\section{ReLigious VieWPOINT}

Different religions and different philosophic frameworks might reach different conclusions about moral dilemmas such as the ones posed by this case. The following discussion will be a Western tradition analysis using catechisms of the Catholic Church as a religious guide and Kantian ethics as a philosophic guide.

Catechism of the Catholic Church \#2278: Discontinuing medical procedures that are burdensome, dangerous, extraordinary, or disproportionate to the expected outcome can be legitimate; it is the refusal of "over-zealous" treatment. Here one does not will to cause death; one's inability to impede it is merely accepted. The decisions should be made by the patient if he is competent and able or, if not, by those legally entitled to act for the patient, whose reasonable will and legitimate interests must always be respected.

There are several guiding principles here. The intent of withdrawal of support must NOT be intent to cause death. Many patients who go through withdrawal of ventilator support continue to breathe on their own. In these cases, the patient should continue to receive ordinary support. When the issue of whether care is ordinary or extraordinary is in dispute, then the decisions should be based on the will of the patient as to the question of when enough is enough. The Church is largely in agreement with the ethical analysis discussed above.

\section{Philosophical VIeWPoint}

The primary question to be resolved from the religious viewpoint is whether the care is ordinary or extraordinary. How do we resolve this question? Philosophy is the search for transcendent truths that guide our answers to questions like this one.

One of the most challenging aspects of a moral philosophy is discovering the foundation upon which it stands. Unlike a religious worldview which can rely on Divine Command Theory and, thus, applicable to all persons, it can be argued that morality is entirely subjective. Yet, many humans express the belief that 
there exists a sense of objectivity to morality that is able to rise above specific individuals and situations. There are actions that people will agree are mandatory to all people in any situation. For example, one ought to save a drowning baby even if the potential savior dislikes babies. Furthermore, this action reaches the moral implication of an 'ought' irrespective of the presence or absence of a legal authority. Although one must render aid, the threat of legal ramifications should be a moot point. It simply will be done.

However clear this feeling of "ought-ness", philosophers throughout our history have struggled to find the metaphysical foundation for the surety of action. It is no secret that there are very few things outside of a human's physical nature that are common to us all. Thus, the foundational principle for moral action is elusive. Kant argued that "moral requirements are based on a standard of rationality dubbed the "Categorical Imperative." 1 Since (supposedly) all humans without brain injuries or defects have the same access to rational thinking, any breach of the Categorical Imperative is irrational and, therefore, immoral. Kant's moral philosophy allows us as rational actors to successfully ground morality universally which creates the necessary "ought-ness". Any action which can rise to an "ought" for any rational actor in any conceivable place, time, and situation is moral. In the particular case of this man, different rational actors would come to different conclusions about the morality of denying care in such a short time span. The relative quickness of the conclusion would necessarily cause it to become irrational and immoral. While it is irresponsible and impossible to forego a conclusion until all humans living can be consulted, it is only until a consensus of care can be reached with all relevant actors that such a decision be reached. It is only when we treat each person as an "end" and not a "means" that we touch true morality.

\section{Practical Viewpoint}

How long should we wait for a consensus? Biology can provide us with a time frame acceptable to everyone. Patients mostly desire to recover from their illnesses. Patients and families have a fear of long, protracted and eventually futile treatments that do not lead to recovery. Although the socialization of health care costs eliminates the financial burden of a long treatment, emotional and opportunity costs remain a burden on the family. In this case the nature of mechanical ventilation provides a landmark that can be used to satisfy everyone involved. Around 14 days of intubation, a tracheostomy is required to prevent permanent damage to the trachea. This is viewed by most families as an escalation of therapy and a significant event. If a patient fails to demonstrate meaningful improvement in whatever process is making life support necessary after 14 days, the likelihood of an eventual meaningful recovery is poor. ${ }^{2}$ In this particular case, if treatment failed to resolve the recurring seizure activity after 14 days, the prognosis would be grim. When the conflict between the health care team and family is due to pessimism from the family, the requirement for tracheostomy can be used as sign post about whether to withdraw care or continue.

The other problem in which the family appears unrealistically optimistic about recovery is more difficult to handle. Although the same 14 day requirement for tracheostomy can be used as a benchmark for making the critical decision, some families want to push on even when the health care team believes further care is futile. When this conflict persists beyond tracheostomy, there may be no attractive options. If the patient has appointed a medical power of attorney, neither an ethics committee nor a court will likely overrule the decision by the legally appointed decision maker. If there is no medical power of attorney, then an ethics committee or court may be consulted to overrule consensus by the family.

\footnotetext{
Author Affiliation: Saad Farooq is a resident in Internal Medicine in the Department of Internal Medicine at TTUHSC. Michael Nugent studies religion and philosophy at the University of Texas in Austin. Gilbert Berdine is a pulmonary physician in the Department of Internal Medicine at TTUHSC.

Received: $11 / 7 / 2013$

Accepted: 1/10/2014

Reviewers: Mike Reagan MD, Kenneth Nugent MD

Published electronically: 1/15/2014

Conflict of Interest Disclosures: None
} 


\section{REFERENCES}

1. Kant Categorical Imperative: http://en.wikisource.org/ wiki/Groundwork_of the_Metaphysics_of_Morals

2. Prognosis and mechanical ventilation:

http://www.ncbi.nlm.nih.gov/pubmed/12771605 